\title{
Novos produtores de média? 0 perfil do produtor de podcasts português
}

\section{New media producers? The profile of the portuguese podcast producer}

\author{
Ruben Martins*, Jorge Vieira* \\ * ISCTE-Instituto Universitário de Lisboa, Centro de Estudos e Investigação de Sociologia
}

Resumo

\begin{abstract}
Com a massificação do formato de podcast (Morgan, 2015), surgiram em Portugal conteúdos áudio criados fora dos circuitos tradicionais das rádios. A acessibilidade desta tecnologia fez com que os produtores de conteúdo a usassem para chegar a uma audiência (Berry, 2006).

A digitalização da rádio e o surgimento da tecnologia de podcasting levou à existência de novos produtores de conteúdos em áudio exclusivamente para a internet. A democratização do meio (Balzen, 2017), possibilitada através da existência de software de baixo custo ou de código aberto, da massificação dos reprodutores portáteis, e das plataformas de online blogging (Berry, 2018) tem levado ao aumento e diversificação dos conteúdos disponíveis.

Para perceber quem são os produtores de podcasts (podcasters) em Portugal foi aplicado um inquérito online (com uma amostra de 92) através do qual foi possível identificar o perfil-tipo do produtor português: homem, com formação superior, sem experiência anterior em rádio, que lança em média um episódio novo do seu formato a cada semana e com uma idade média de 31 anos.

Este artigo apresenta ainda a caracterização do mercado português de podcasts independentes no momento imediatamente anterior à realização do inquérito (Setembro de 2018), tendo sido registado a existência de uma centena de formatos, 77 dos quais ativos aquando do levantamento. Eram as categorias de "Comédia", "TV e Cinema" e "Desporto e lazer" que apresentavam mais episódios disponíveis.
\end{abstract}

Palavras-chave: Podcast, Rádio, Novos Media.

Abstract

With the massification of the podcast format (Morgan, 2015), audio content created outside of traditional radio circuits emerged in Portugal. The accessibility of this technology made content producers use it to reach an audience (Berry, 2006).

The digitalization of radio and the emergence of podcasting technology has led to the existence of new producers of audio content exclusively for the internet. The democratization of the medium (Balzen, 2017), made possible through the existence of low cost and open-source software, the massification of portable players, and blogging platforms (Berry, 2018) has led to an increase and diversification of the available content.

In order to understand who are the podcast producers in Portugal, an online survey (with a sample of 92 responses) was applied through which it was possible to identify the type profile of the Portuguese producer: man, with higher education, without previous experience in radio, who launches on average a new episode of its format every week and with an average age of 31 years.

This article also presents the characterization of the portuguese market of independent podcasts in the moment immediately before the survey (September 2018), having recorded the existence of a hundred formats, 77 of which were active at the time of the survey. It was the categories of "Comedy", "TV and Cinema" and "Sport and leisure" that had the most episodes available.

Keywords: Podcast, Radio, New Media.

\section{Introdução: Podcast, podcasters e podcasting}

No contexto da digitalização da rádio (Cordeiro, 2014) a internet passou a ser utilizada como extensão da realidade do áudio analógico e surgiram novas perspetivas para a sua utilização. As webradios, que 
consistem em emissões lineares de rádio sem necessidade de recurso à transmissão por via herteziana ou satélite, usando uma sequência de valores de sequência sonora representado por bits (Portela, 2011: 13) foram precursoras da utilização do áudio na internet. O primeiro registo de uma rádio online data de 1993, tendo como nome "Internet Talk Radio". Este foi um projeto da autoria do norte-americano Carl Malamud¹. Em Portugal, a internet afirmou-se como complementar às emissões em FM (Cordeiro, 2014) aproveitando a fiabilidade técnica, que lhe permite emitir sem interferências e amplificar a cobertura existente por via terrestre. Esta amplificação permitiu a chegada de novos conteúdos aos que estão ligados através da internet.

Dados da ANACOM², referentes ao ano de 2018, mostram que a penetração da internet nos lares portugueses é já bastante elevada, chegando a 76,3\% das famílias ${ }^{3}$. Ainda assim, Portugal é o terceiro país com uma percentagem mais baixa de lares com acesso a banda larga na União Europeia a 28, havendo apenas registos inferiores para a Grécia (76\%) e Bulgária (71\%) 4 .

Nos primeiros seis meses de 2019, os dados da Bareme Rádio ${ }^{5}$ da Marktest apontaram para um recorde de escuta de rádio através da internet em Portugal. Um total de 1 milhão e 848 mil residentes em Portugal Continental com 15 ou mais anos (21,6\% do universo) afirmaram que costumam ouvir rádio através da internet ${ }^{6}$. A Marktest afirma que são as pessoas com idades entre os 25 e os 34 anos aqueles que mais consomem rádio pela internet, algo que $38,5 \%$ dos inquiridos daquela faixa etária afirma fazer. Pelo lado oposto, apenas três em cada 100 indivíduos com mais de 64 anos ouve rádio na internet.

Para além da utilização da internet para fins de transmissão da emissão linear, a rádio aproveitou os recursos online como forma de repositório on-demand (Portela, 2011: pp. 18) e, mais tarde, através da produção de conteúdo áudio especificamente pensado e adaptado à realidade do consumo online.

"O podcast torna possível a cada um seguir o impulso individual e produzir o seu próprio espaço radiofónico, que assim se apresenta fragmentado nas suas audiências e endereça uma abrangente amplitude de interesses, criando nichos de públicos bastante específicos"(Portela, 2011: 18).

Um dos fenómenos mais relevantes do contexto digitalização da rádio são os podcasts, desenvolvidos nos primeiros anos do milénio por Adam Curry e Dave Winer (Crofts et al., 2005), apesar de ainda ser alvo de disputa a criação da tecnologia 7.

A adesão do público em combinação com a massificação dos leitores portáteis de reprodução de música, como iPods e smartphones, a existência de software de código-aberto, gratuito ou com preços muito reduzidos, e o crescimento das plataformas de blogging foram fatores que levaram a que se criassem bases para uma "nova era de ouro do áudio" (Berry, 2018: 20). A todos estes fatores podemos juntar a facilidade

\footnotetext{
${ }^{1}$ in Uma breve história da rádio pela internet (2016), por Jamie Ashbrook (tradução livre do autor: "A Brief History of Internet Radio"). Consultado a 4 de Setembro de 2019. Disponível em: https://radio.co/blog/a-brief-history-of-internetradio

${ }^{2}$ Autoridade Nacional das Comunicações

${ }^{3}$ Conforme dados da ANACOM disponíveis em: https://www.anacom.pt/render.jsp?contentId=1472382. Consultado a 4 de Setembro de 2019.

${ }^{4}$ Dados do Eurostat consultados a 4 de Setembro de 2019 disponíveis em: https://ec.europa.eu/eurostat/web/productsdatasets/product?code=ISOC_CI_IN_H.

${ }^{5}$ Estudo de referência para o mercado de rádio em Portugal, responsabilidade da Marktest.

${ }^{6}$ Dados da Markest disponíveis em: https://www.marktest.com/wap/a/n/id 2544.aspx

7 Conforme consultado em: https://www.cnet.com/news/who-invented-podcasting-who-cares/
} 
de acesso à internet e a cada vez maior capacidade de transferência de dados, por custos reduzidos e a velocidades elevadas e o maior interesse por parte dos criadores de conteúdos e dos próximos utilizadores no formato e na tecnologia.

Os próprios meios de comunicação social adotaram esta tecnologia para chegar a novas audiências. Refere Reis (2018) que "os jornais usam o podcast como uma estratégia de valorização e afirmação do jornal enquanto opinion maker". As próprias rádios empregam o podcast como tecnologia de disponibilização dos seus conteúdos on-demand.

No seu geral, os podcasts revelaram-se com uma tecnologia "profundamente democrática" (Balzen, 2017) ao permitir a criação e distribuição de conteúdo de forma acessível, sem necessidade de deter recursos técnicos, legais e monetários característicos da produção profissional de conteúdos. Ao mesmo tempo, os perfis traçados sobre a realidade dos produtores de podcasts mostram que continuam a ser aqueles que têm um maior grau de escolaridade a utilizar esta tecnologia para produzir conteúdo (Markman \& Sawyer, 2014). Também a nível de género há um desequilíbrio entre os produtores de conteúdos, favorável ao sexo masculino.

Dados do Digital News Report de $2019^{8}$, com base em dois mil inquéritos, mostram que $34 \%$ dos inquiridos em Portugal tinham ouvido um podcast no mês anterior ao estudo, uma das mais elevadas taxas entre os países abordados no relatório.

Necessária é a distinção entre duas facetas que partilham a mesma terminologia: o podcastcomo tecnologia e a do podcast como conteúdo. O podcast como tecnologia surge primeiro e representa "um mecanismo de distribuição de ficheiros MP3 através da internet"' (Llinares et al., 2018: 5).

Deste mecanismo surge um complexo sistema de distribuição assente num sistema de subscrição, geralmente gratuita, que avisa e distribui aos subscritores cada novo episódio disponível através de várias plataformas dedicadas, como o Spotify ou o Apple Podcasts. O podcast como conteúdo assenta as suas bases na produção de conteúdos dedicados a esta tecnologia e as potencialidades que esta oferece (Berry, 2018: 15).

Um conteúdo distribuído através da tecnologia de podcast pode não ser de origem um podcast (Vanassi, 2007), exemplos disso estão, por exemplo, na distribuição de um determinado programa de rádio ou um áudio de um programa de televisão através desta tecnologia.

\section{Dados de estudos anteriores}

Como forma de colmatar uma lacuna existente no campo de conhecimento científico, o único mapeamento anteriormente realizado à realidade do podcasting português data de 2006 por Luís Bonixe. À época, o investigador utilizou como universo "os podcastsportugueses registados nos diretórios Lusocast e Cotonete [ambos desativados atualmente] entre os dias 1 e 14 de Março de 2006 e que nessa altura tivessem sido atualizados pelo menos uma vez nos últimos dois meses. Foram excluídos os podcasts cuja autoria fosse de empresas de comunicação social" (Bonixe, 2006).

\footnotetext{
8 Retrato de Portugal por Ana Pinto-Martinho, Miguel Paisana e Gustavo Cardoso do ISCTE- IUL. Disponível em https://reutersinstitute.politics.ox.ac.uk/sites/default/files/2019-06/DNR_2019_FINAL_0.pdf.

${ }^{9}$ Tradução livre do autor. "Podcasting is a delivery mechanism, a means of distributing MP3 audio files across the internet". (Llinares et al., 2018, pp. 5)
} 
Foram então catalogados 31 podcasts que correspondiam a estes requisitos e a quem foram enviados inquéritos. Dos 17 autores que responderam ao pedido do investigador foi possível tirar conclusões sobre o género e idade dos produtores, que na sua grande maioria eram homens (73\%) e menores de 30 anos (76,19\%). 78\% tinham formação superior, num panorama semelhante ao mercado norte-americano descrito por Markman e Sawyer em 2014.

Em Portugal, a maioria dos formatos era produzido por uma só pessoa (52\%), atualizados semanalmente e tinham convidados. Os produtores independentes inquiridos viam o podcast como "uma nova forma de disponibilizar outros conteúdos que não se enquadram nas 'formatações' das rádios" (Bonixe, 2006).

Em relação à realidade norte-americana, a produção independente de podcasts foi explorada no Journal of Radio \& Audio Media em dois artigos: "Porquê 'Pod'? Novas explicações das motivações do podcasting independente" (Markman \& Sawyer, 2014) e "Fazer rádio, fazer amigos e divertir-se: Explorar as motivações dos podcasters independentes" (Markman, 2011).

O primeiro artigo citado mostra que os "resultados de um inquérito online dizem que o podcasting continua a ser dominado por homens com mais de 30 anos, com formação superior, e que não vivem do podcast a tempo inteiro" (Markman \& Sawyer, 2014:20). Há também uma tendência de procura de novos modelos de negócio que permitam aos produtores de conteúdo desenvolver conteúdo a tempo inteiro, uma vez que a maioria o faz de forma complementar a outra atividade profissional. Estas conclusões foram feitas a partir de 120 inquéritos online, numa base inicial de 355 formatos catalogados. Dos inquiridos, 45\% dos podcasters independentes tinha uma experiência anterior na área dos media.

Em relação ao artigo de (Markman, 2011), os princípios de estudo foram semelhantes. Destaca-se neste estudo a utilização do método de bola de neve, "usado para localizar, aceder e envolver pessoas de populações específicas nos casos em que o investigador antecipa dificuldades em criar uma amostra representativa da população da pesquisa" (Cohen e Arieli, 2011: 426-427). A resposta à questão de partida - O que te fez começar a fazer podcasts? - das seis categorias previamente definidas, as respostas mais frequentes foram "as motivações pela própria tecnologia/media, conteúdo e motivos pessoais" (Markam, 2011:555).

\section{Metodologia}

Para a realização deste estudo mobilizou-se a seguinte metodologia a dois momentos: um mapeamento de diagnóstico do panorama nacional e um inquérito dirigido aos podcasters e com um intuito de caracterização, Desta forma foi primeiramente realizado um exercício de levantamento de podcasts nacionais. Entre 27 de Agosto e 19 de Setembro de 2018 foram mapeados todos os formatos disponíveis na loja portuguesa da iTunes (propriedade da Apple), que não fossem propriedade ou produção de um órgão de comunicação social ou de uma marca.

Como requisito, os formatos tinham também de ser falados em língua portuguesa, produzidos em Portugal, e figurar entre os 200 formatos mais bem classificados no "Top de programas" de uma das 16 categorias utilizadas pela plataforma para agregar conteúdos de acordo com a sua temática ${ }^{10}$ : Arte, Negócios, Comédia,

\footnotetext{
${ }^{10}$ São os próprios autores que definem em que categoria se querem incluir, tendo em conta as categorias disponíveis.
} 
Ensino, Jogos e passatempos, Governo e organizações, Saúde, Infantil e familiar, Música, Notícias e Política Religião e espiritualidades, Ciência e medicina, Sociedade e cultura, Desporto e lazer, Tecnologia, TV e cinema.

Nos formatos de "Música" não foram considerados os programas sem qualquer registo oral, excluindo à partida complicações de disco-jóqueis e programas de playlist. Também não foram considerados na análise os programas que são distribuídos através de uma ou mais emissoras de rádio, mesmo que sejam considerados pelos produtores como "formatos de autor".

Ainda assim, este mapeamento dos podcasts independentes da iTunes Store Portugal não representa a totalidade de formatos de produção independente existente em Portugal, ficando excluídos da análise programas que só contam com presença noutras plataformas ou que contam com audiências residuais.

Passando para o inquérito online, esta abordagem quantitativa privilegiou perguntas maioritariamente fechadas, e seguiu na construção do seu guião alguns conceitos e molduras teóricas previamente utilizados nas pesquisas de Bonie (2006) em Portugal e de Markaman e Sawyer (2014) nos Estados Unidos da América. Durante um período de três meses foi pedido aos podcasters portugueses que respondessem a um inquérito online através da plataforma Google Forms, cujo formulário se encontra disponível de forma integral nos anexos.

O inquérito foi constituído por 38 perguntas procurando avaliar parâmetros sociodemográficos dos podcasters e os próprios formatos que produzem ou em que participam.

Entre 23 de Outubro de 2018 e 1 de Fevereiro de 2019 o inquérito esteve aberto a respostas, sendo prérequisito amostral para poder participar: "ser autor, produtor, colaborador, editor ou membro do painel de um programa áudio disponibilizado em formato de podcast e produzido em Portugal", conforme enunciado aquando da abertura do inquérito.

No que concerne à disseminação, o formulário foi enviado diretamente para alguns produtores de podcasts e disponibilizado em dois grupos de Facebook que agregam podcasters portugueses: "Podcast(in)g Portugal" e "Podcasters Portugal". Estes grupos contam com 93 e 74 membros respetivamente (a grande maioria dos podcasters pertencia aos dois grupos) à data da publicação. $O$ inquérito foi também partilhado na rede social Twitter via "Portcasts Portugal", um perfil para a "promoção de podcasts portugueses" com 509 seguidores, a 12 de Junho de 2019.

Eram objetivos do estudo:

- Caracterizar o podcaster português.

- Perceber motivações para a sua produção.

Foram obtidas 92 respostas válidas.

\section{Perfil do podcaster português}

A maioria dos podcasters portugueses que responderam ao inquérito revelou não ter experiência de rádio anterior à produção do podcast (62\%). Para quem afirmou ter tido essa experiência anterior, apresentavam valores relevantes as respostas mencionando a experiência obtida numa rádio universitária (com 18 respostas) e rádio local (com 14). 
Quase metade dos podcasters afirma participar ou já ter participado regularmente em mais de um formato $(44,6 \%)$ e 10,9\% admite participar ou ter participado em mais de três formatos.

Em relação ao género, o podcaster português é maioritariamente homem $(76,1 \%)$.

No que toca à variável idade, a média do podcaster português situa-se nos 30,8 anos, num intervalo com respostas entre os 17 e os 49 anos, sendo as idades mais frequentes os 23 (com nove respostas) e os 35 (com sete respostas). A mediana situa-se nos 31 anos.

Gráfico 1: Podcasters portugueses por idade. Elaboração própria.

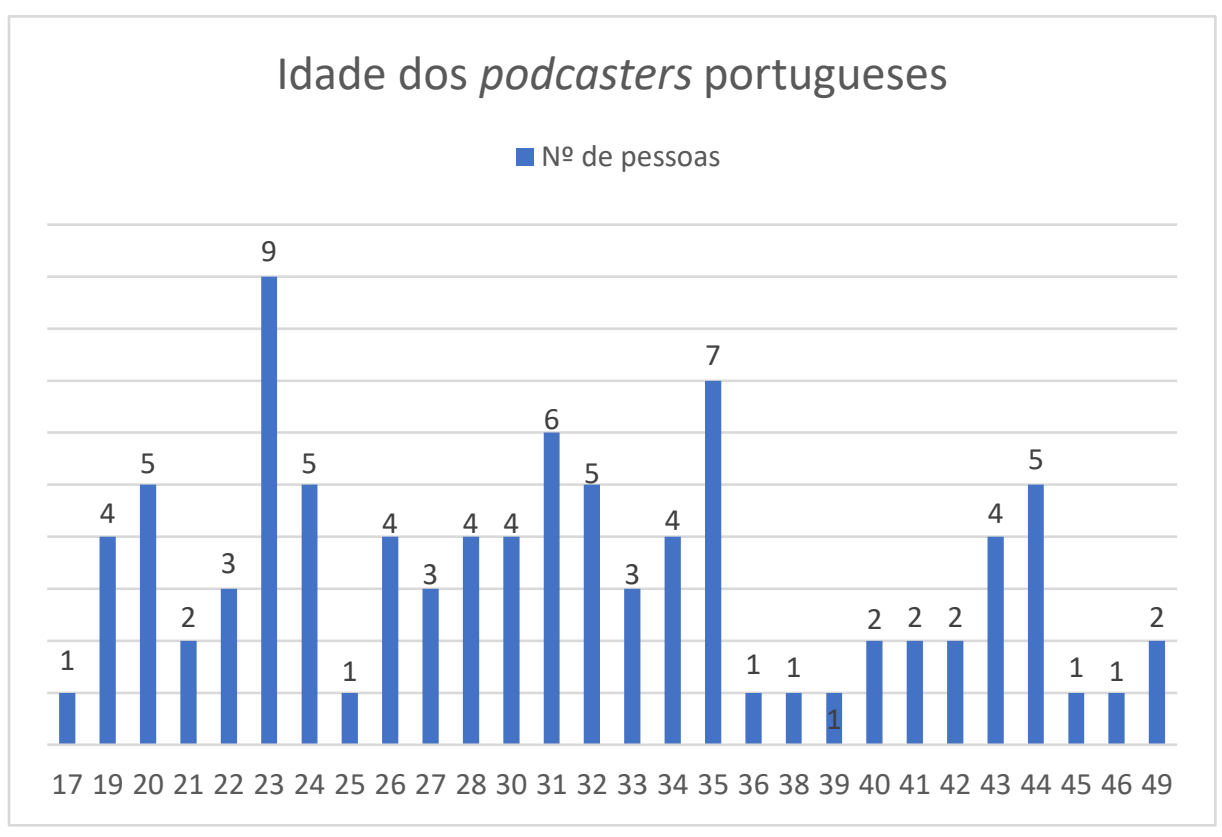

À data do inquérito, existiam dois podcasters independentes exclusivamente dedicados à produção de conteúdos em áudio para a internet. Grande parte dessa explicação deve-se ao facto de a grande maioria não dispor de publicidade: 90,2\% não apresenta qualquer parceira remunerada sob forma de publicidade ou qualquer outro tipo de apoio monetário. Em relação à profissão do podcasterportuguês, das 90 respostas válidas nesta pergunta, é possível identificar duas ocupações que se repetem várias vezes: estudante (com 16 referências) e jornalista (com 12 referências). 
Tabela 1: Profissão dos podcasters portugueses (na tabela apenas aparecem ocupações com mais de duas referências). Elaboração própria.

\begin{tabular}{|c|c|}
\hline Profissão & Número de podcasters \\
\hline Estudante & 16 \\
\hline Jornalista & 12 \\
\hline Humorista & 4 \\
\hline Consultor & 4 \\
\hline Animador de Rádio & 3 \\
\hline Produtor de Conteúdos & 2 \\
\hline Gestor & 2 \\
\hline Fotógrafo & 2 \\
\hline Especialista em agência de & 2 \\
\hline comunicação & 2 \\
\hline Assessor de imprensa & \\
\hline
\end{tabular}

Em relação à escolaridade do podcaster, a resposta mais frequente é a opção licenciatura. Daqueles que apenas dizem ter o ensino secundário completo, é comum apresentarem "estudante" como ocupação principal. Há ainda $26,1 \%$ que diz ter concluído um mestrado.

Tabela 2 - Escolaridade dos podcasters portugueses. Elaboração própria.

\begin{tabular}{|l|c|}
\hline \multicolumn{1}{|c|}{ Escolaridade } & Número de respostas \\
\hline Menos que o 9.0 ano completo & 0 \\
\hline Ensino Básico - $\mathbf{9 . 0}$ ano completo & 0 \\
\hline Ensino Secundário - 12.0 ano completo & 29 \\
\hline Licenciatura & 38 \\
\hline Mestrado & 24 \\
\hline Doutoramento & 1 \\
\hline
\end{tabular}

Comparado com o perfil dos níveis educacionais da população portuguesa no ano de 2018 , onde $18,7 \%$ dos portugueses tinham um grau académico de ensino superior ${ }^{11}$, observamos que a taxa de podcasters com edução superior corresponde a 68,5\%, mostrando que, apesar de mais democrática do que a rádio convencional e os outros meios tradicionais, esta tecnologia continua a ser utilizada para produção de conteúdos de forma maioritária por aqueles que mais estudam.

\footnotetext{
${ }^{11}$ Conforme dados agregados pelo portal Pordata disponíveis em:

https://www.pordata.pt/Portugal/Popula\%c3\%a7\%c3\%a3o+residente+com+15+e+mais+anos+por+n\%c3\%advel+de +escolaridade+completo+mais+elevado+(percentagem)-884
} 
Quando questionados sobre se colocariam a produção do seu podcastnum currículo a entregar num possível empregador, $71(81,5 \%)$ disseram que sim e seis $(6,5 \%)$ optaram pela resposta "não sei". Há ainda 59 $(64,1 \%)$ produtores que afirmam que, se pudessem, teriam a produção de podcasts como "atividade permanente, única e remunerada".

A maioria dos produtores de podcasts em Portugal considera que usa "material profissional" na produção do podcast $(70,1 \%)$, e a maioria grava em casa $(59,3 \%)$. Há também 22 podcasters que dizem usar estúdios que pertencem a uma rádio. $58 \%$ dos podcasters diz ter lançado o seu podcastnum dos 48 meses anteriores ao estudo.

No que concerne às motivações para se iniciar a produção de um conteúdo para distribuição através da tecnologia de podcast, a resposta mais comum para justificar é "para se divertir" (89,1\%).

Há ainda $82,7 \%$ que dizem que produzir o podcast é "uma forma de se realizar pessoalmente" e 77,2\% recusam que tenham sido "motivos financeiros" a incentivá-los à produção do podcast. Quase $70 \%$ (69,6\%) afirma que o objetivo de lançar o programa não partiu de uma vontade de experimentar algo semelhante à rádio. Metade dos produtores acredita que o seu formato "é algo único no panorama português".

No que toca à regularidade dos formatos produzidos, a grande maioria tem uma periodicidade semanal (62\%), ainda assim 40,2\% diz ter dificuldade em cumprir com a periodicidade pré-determinada. Há também $19,6 \%$ dos podcasters inquiridos que declarou já ter pensado acabar com a produção do podcast.

À data, o iTunes (atual Apple Podcasts) era a plataforma preferencial para distribuição dos podcasts. 83,7\% dos produtores afirmavam ter o conteúdo disponível naquela plataforma. O Spotifyera a segunda plataforma (53,3\%) para os podcasters portugueses.

Para divulgar o podcast a maioria dos produtores $(80,4 \%)$ tinha uma página do podcast na rede social Facebook. Quase metade $(49,4 \%)$ tinham página de Instagram, a mesma percentagem para os perfis no Twitter. $76,6 \%$ diz que tem um e-mail público para favorecer o contacto com os ouvintes.

Em relação à tipologia, a grande maioria dos inquiridos $(80,4 \%)$ produzia formatos de entrevista (37 formatos) ou programas de debate em painel aberto com intervenientes diferentes nos vários episódios ( 37 formatos).

Sobre audiências, $11,1 \%$ diz não ter objetivos neste tópico. Há ainda $45,6 \%$ dos podcasters que valoriza de forma negativa as audiências que então tinha em relação às expectativas iniciais aquando do começo da produção. Quando questionados sobre o valor ideal de ouvintes, o valor mais apresentado era o de mil pessoas. Este número foi mencionado por 17 produtores inquiridos.

Em relação à disposição para incluir publicidade nos seus formatos, 56,3\% diz que teria disponibilidade para aceitar patrocinadores ao formato, contra $17,2 \%$ que o rejeita. Como principal razão para rejeitar a presença de anunciantes está a não intenção de fazer dinheiro com o podcast (justificação apresentada por dez podcasters). Há ainda nove podcasters que receiam perder a independência com a presença de anunciantes nos seus formatos.

\section{A realidade do podcast independente português}

Para completar a análise ao mercado português, contextualizando os dados apresentados com dados sobre os conteúdos disponíveis, entre 27 de Agosto e 19 de Setembro de 2018 foi realizado um primeiro 
mapeamento dos formatos de podcast independentes (não pertencentes a órgãos de comunicação social) existentes em Portugal. A fragmentação das plataformas de distribuição de podcasts obrigou à imposição de limites ao universo em análise, tendo por isso sido limitado à loja portuguesa da iTunes. Esta plataforma da Apple foi lançada a 9 de Janeiro de 2001, tendo começado a ser compatível com a tecnologia de podcasting em Junho de 2005, sendo apontado também como um fator de massificação do formato:

"O iTunes da Apple ajudou a tornar popular o podcasting ao incorporar em suas versões mais recentes softwares agregadores que organizam os conteúdos dos podcasts, além de disponibilizar um catálogo dos podcasts mais populares" (Castro, 2005: 5-6)

Em Junho de 2018, a Apple anunciava na Conferência Mundial de Programadores da Apple (WWDC) a marca de 550 mil podcasts ativos na plataforma e um total de 18.5 milhões de episódios.

O único mapeamento anteriormente realizado à realidade do podcasting português data de 2006 e é da autoria do professor e investigador Luís Bonixe. À época, o investigador utilizou catalogou 31 podcasts que correspondiam ao pré-requisitos anteriormente apresentados.

\section{Resultados}

Foram encontrados 100 formatos que se enquadravam nas condições de análise requeridas e detalhadas na metodologia. Este número representa $3,1 \%$ dos 3200 podcasts que correspondem ao universo dos podcasts presentes entre os 200 melhores classificados de entre as 16 categorias da loja portuguesa do iTunes.

De acordo com as categorias a maioria dos formatos de podcast independente classifica-se como "Comédia" (20 programas) e "Desporto e Lazer" (16 programas). No ponto oposto, as categorias onde não se encontram formatos independentes nas tabelas dos melhores classificados são "Governo e Organizações" e "Infantil e Familiar".

Tabela 3: Número de formatos por categoria nos tops da loja portuguesa da iTunes Store e número de formatos ativos. Elaboração própria.

\begin{tabular}{|l|c|c|c|}
\hline \multicolumn{1}{|c|}{ Categoria } & Número de formatos & Formatos ativos & $\begin{array}{c}\text { Percentagem em } \\
\text { produção (ativos por } \\
\text { total de formatos) }\end{array}$ \\
\hline Comédia & 20 & 13 & $65 \%$ \\
\hline Desporto e Lazer & 16 & 12 & $75 \%$ \\
\hline Sociedade e Cultura & 15 & 9 & $60 \%$ \\
\hline TV e Cinema & 12 & 8 & $66,66 \%$ \\
\hline Tecnologia & 8 & 2 & $25 \%$ \\
\hline
\end{tabular}




\begin{tabular}{|c|c|c|c|}
\hline Arte & 7 & 4 & $57,14 \%$ \\
\hline Música & 5 & 3 & $60 \%$ \\
\hline Jogos e passatempos & 4 & 2 & $50 \%$ \\
\hline Saúde & 4 & 3 & $75 \%$ \\
\hline Negócios & 3 & 1 & $33,33 \%$ \\
\hline Ensino & 2 & 2 & $100 \%$ \\
\hline Notícias e Política & 2 & 1 & $50 \%$ \\
\hline $\begin{array}{l}\text { Religião e } \\
\text { Espiritualidades }\end{array}$ & 1 & 1 & $100 \%$ \\
\hline Ciência e Medicina & 1 & 1 & $100 \%$ \\
\hline $\begin{array}{l}\text { Governo e } \\
\text { Organizações }\end{array}$ & 0 & 0 & - \\
\hline Infantil e Familiar & 0 & 0 & - \\
\hline TOTAL & 100 & 62 & $62 \%$ \\
\hline
\end{tabular}

\section{Podcasts ativos e média de episódios:}

Para análise do número de podcasts que continuam a ser atualizados com novos episódios, foram considerados "podcasts ativos" os formatos anteriormente descritos que tinham um episódio posterior a 1 de Junho de 2018 e cujo último episódio não tinha uma menção ao fim do programa no título ou descrição. Apesar de alguns formatos se manterem no top de programas, nem todos os formatos analisados se encontravam "ativos".

A média de episódios dos formatos inativos situava-se, a 19 de Setembro de 2018, nos 33 episódios. No entanto os números são bastante variáveis entre cada formato, uma vez que há conteúdos que deixam de ser atualizados depois de lançado o primeiro episódio - são os casos de "Quem não tem cão, casa com gato" e "Aurora Boreal". No ponto oposto, "Os melhores adeptos" produziram, entre 2013 e 2018, 152 episódios antes de se terem tornado "inativos", apesar de oficialmente não terem posto um fim ao formato. Nos formatos ativos a média do número de episódios situa-se nos 57 episódios.

A categoria que mais conteúdos e episódios tem disponíveis é a de "Comédia", com 20 formatos e 972 episódios disponíveis. O peso desta categoria no universo de podcasts independentes explica-se, em parte, com o facto de vários humoristas, com presença mediática, utilizarem a tecnologia de podcasting para chegar ao seu público. A 24 de Setembro de 2018, dos cinco formatos independentes mais bem cotados, três eram de humoristas (Pedro Teixeira da Mota, Salvador Martinha e o coletivo Bruno Nogueira, Filipe Melo e Nuno Markl), um era de um apresentador de televisão, apesar de estar integrado na categoria de "Comédia" (Rui Unas) e o outro formato pertencia ao colunista e comentador Daniel Oliveira. 
Gráfico 2: Número de episódios disponíveis por categoria nos podcasts independentes na loja portuguesa da iTunes Store (inclui formatos ativos e não ativos). Elaboração própria.

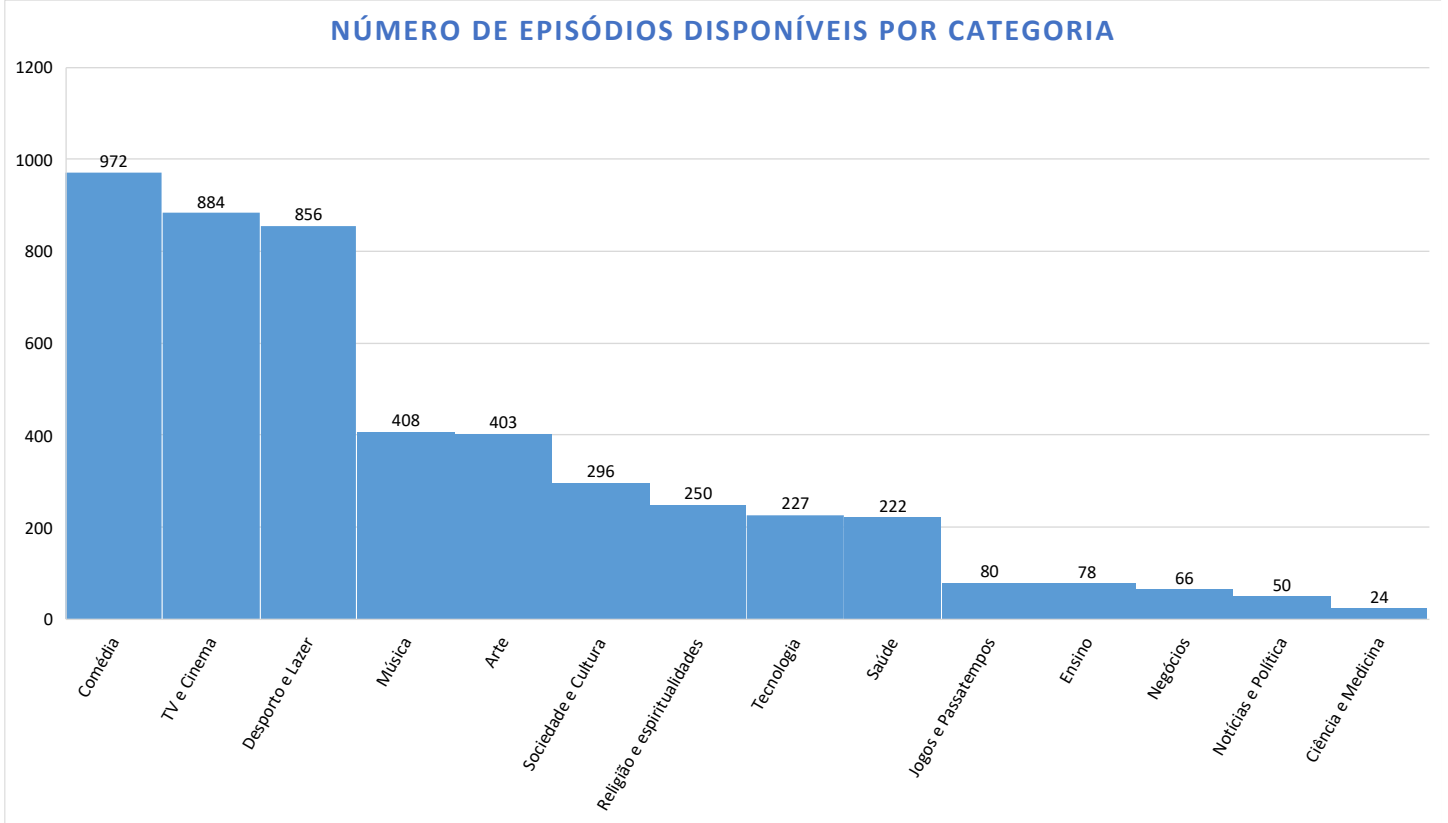

Há ainda dois tipos de conteúdos que, pela relevância no mercado, se destacam dos restantes: "TV e Cinema" e "Desporto e Lazer".

Olhando para o panorama de conteúdos de desporto verifica-se uma predominância dos formatos sobre futebol em detrimento dos outros desportos. Doze dos 16 formatos são sobre esta modalidade, destes cinco falam sobre vários clubes, dois deles apenas falam sobre a atualidade do Sporting Clube de Portugal e outros dois do Sport Lisboa e Benfica e um formato sobre o Futebol Clube do Porto. Apesar de debaterem a atualidade dos vários emblemas nenhum destes formatos conta com o apoio oficial dos clubes. Há ainda outro formato sobre dados da história do futebol e ainda outro programa sobre corrupção no futebol português, nomeadamente sobre o caso conhecido publicamente como "Caso dos e-mails do Benfica". Em relação a outros desportos há ainda dois podcasts sobre Basquetebol, um sobre Fitness e outro sobre Artes Marciais Mistas (MMA).

Tabela 4: Número de formatos de desporto por tema e número de episódios. Elaboração própria.

\begin{tabular}{|c|c|c|}
\hline Formatos desporto & Número de Formatos & Número de episódios \\
\hline Futebol - Vários Clubes & 5 & 310 \\
\hline Futebol - Sporting Clube de Portugal & 2 & 137 \\
\hline Futebol - Sport Lisboa e Benfica & 2 & 43 \\
\hline Futebol - Futebol Clube do Porto & 1 & 73 \\
\hline Futebol - História & 1 & 47 \\
\hline Futebol - Corrupção & 1 & 26 \\
\hline
\end{tabular}




\begin{tabular}{|c|c|c|}
\hline Basquetebol & 2 & 206 \\
\hline Fitness & 1 & 10 \\
\hline Artes Marcais Mistas & 1 & 73 \\
\hline
\end{tabular}

O formato independente com mais episódios chama-se "Rádio Defusão". O formato tem autoria de Fábio Vieira Fernandes e Catarina Nunes e desde 2010 já lançou 277 episódios. Este formato está integrado na categoria "música", tem site próprio e presença nas redes sociais online. Algumas das temporadas do formato foram produzidas para a rádio universitária da Escola Superior de Comunicação Social (ESCS FM) e para a, entretanto extinta, rádio online Antena 3 Rock, do grupo Rádio e Televisão de Portugal (RTP).

\section{Conclusões}

A realidade dos produtores de podcasts portugueses continua a ser desfasada do perfil médio da população. Apesar de se afirmar como uma alternativa de comunicação acessível, a tecnologia de podcasting é utilizada de forma mais evidente pela franja da população com um maior nível de educação.

Com mais de uma década de amadurecimento, a tecnologia continua a poder ser apresentada como um formato de nicho, com poucos conteúdos originais disponíveis, produzidos por produtores independentes. Este dado pode ser justificado com a larga maioria dos produtores que não aufere receitas diretas com os conteúdos que produz, sendo sinal de um mercado português com pouco valor comercial e pouco interesse por parte das marcas em fazer investimento publicitário nos conteúdos distribuídos através desta tecnologia. A insatisfação com as audiências, face às expectativas inicialmente criadas, afeta quase metade do universo de podcasters portugueses inquiridos $(45,6 \%)$, levando ao fim prematuro de alguns conteúdos depois de poucas semanas de produção.

Os próprios conteúdos produzidos acabam por ser diferenciar pouco dos modelos produzidos pelas rádios convencionais de emissão linear. No universo de podcasts portugueses destacam-se os conteúdos de entrevista e debate/conversa entre vários intervenientes a cada semana.

Podemos dizer que, de acordo com os resultados do inquérito, o podcaster português tipo é homem, licenciado, sem experiência anterior em rádio, que lança um episódio novo do seu formato a cada semana e com uma idade média de 31 anos. Este é um resultado que se enquadra nos estudos comparáveis supracitados.

Estes produtores independentes encontram na tecnologia de podcast um novo canal disponível para chegar a uma determinada audiência, no entanto essa mesma audiência transita de outras plataformas, uma vez que entre os programas mais populares da loja portuguesa do iTunes estavam formatos pertencentes a figuras com presença mediática regular noutros meios, nomeadamente na televisão ou rádio convencional de emissão linear.

Em resumo:

- Três em cada quatro produtores de podcasts em Portugal são do sexo masculino.

- Quase quatro em cada dez podcasters portugueses fez rádio antes de lançar o podcast.

- Quase metade dos podcasters diz que já participou em mais do que um podcast.

- Sete em cada dez podcasters diz que, se pudesse, vivia só de fazer o podcast. 
- O motivo mais apresentado para se lançar um podcast é "para me divertir".

- Oito em cada dez podcasters considera o podcast como currículo se tivesse de entregar um CV hoje.

- A maioria dos autores dos formatos têm uma licenciatura concluída (44\%).

Apesar deste perfil, que acaba por ser comum aos vários estudos comparáveis, a valorização que cada produtor faz ao seu conteúdo acaba por ser relativa, uma vez que cada pessoa tem uma noção diferente daquilo que representa o sucesso do seu formato (para uns poderá ser as audiências, para outros o retorno financeiro, ou até o feedback dos fãs e/ou subscritores). No entanto, não é de descartar a tendência de paragem de produção de conteúdos sem qualquer aviso prévio, e a incapacidade assumida por $40,2 \%$ de assegurar a periodicidade de produção pré-determinada.

Este inquérito abrangeu uma boa parte dos podcasters portugueses independentes de então, uma vez que o levantamento da realidade portuguesa foi realizado na antecâmara da realização do inquérito e dava conta da existência de 100 podcasts, de acordo com a metodologia anteriormente já discriminada.

Fazendo uma possível leitura longitudinal, comparativamente com o estudo prévio de Luís Bonixe (2006), podemos observar uma evolução positiva no número de produtores de conteúdos, associado a uma maior popularidade do formato. No entanto, o aumento do número de conteúdos produzidos não deturpou outros indicadores que continuam alinhados com os dados recolhidos por Bonixe treze anos antes: o produtor continua a ser de forma maioritária homem, representando uma percentagem semelhante à detetada por Bonixe em 2006 (73\%), e tem, maioritariamente, formação superior, numa percentagem desfasada e muito superior ao perfil da população portuguesa. Ou seja, a especificidade social da produção de podcasting em Portugal manteve-se ao longo destes anos.

A publicação de um novo episódio a cada semana continua a ser a periodicidade mais comum no panorama do podcasting português (62\% em 2019 contra 64\% em 2006). No que toca a motivações para a criação de conteúdo para distribuição nesta tecnologia, Bonixe detalha que a resposta mais frequente é que o podcast representa "uma oportunidade de expressão" (77\%), no levantamento realizado em 2019 a questão é colocada de forma diferente, no entanto, a maioria dos podcasters inquiridos diz ter motivações de entretenimento na elaboração dos conteúdos. Em ponto oposto, e de forma similar ao levantamento de Luís Bonixe, poucos são os que apresentam motivos comerciais para lançar o podcast, mostrando a ainda poupa maturidade existente no que toca ao mercado comercial de podcasts em Portugal.

Apesar da diferença no que toda à dimensão dos mercados, o perfil do produtor de podcast em Portugal tem uma elevada similitude com a realidade retratada nos Estados Unidos da América por Markman (2015) ou em Espanha por Antunes et. al. (2018), mostrando que o próprio produtor de conteúdos acaba por ter um estilo padrão comum entre mercados, apesar das várias diferenças intrínsecas à própria dimensão do meio nos respetivos países, não analisadas neste artigo. 


\section{Bibliografia:}

Antunes, M. e Salaverría, R. (2018). PodcastSpain: análisis de los podcasts de audio más populares en iTunes de España. Libro de Comunicaciones del VI Congreso Internacional de la AE-IC 'Comunicación y Conocimiento'. pp. 1753-1770

Balzen, R. (2017). Podcasting is THE Democratic Medium. Discover Pods. https://discoverpods.com/podcasting-democratic-medium/

Berry, R. (2006) 'Will the iPod kill the radio star? Profiling podcasting as radio'. Convergence: The International Journal of Research into New Media Technologies, 12(2), pp. 143-162.

Berry, R. (2018) 'Just because you play a guitar and are from Nashville doesn't mean you are a country singer': The Emergence of Medium Identifities in Podcast. Em Llinares, D., Fox, N. e Berry, R (Ed.), Podcasting. New Aural Cultures and Digital Media. (Edição, volume, pp. 15-33). London: Palgrave Macmillan

Bonixe, L. (2006). Um olhar sobre podcasting português. http://radioejornalismo.blogspot.com/2006/05/um-olhar-sobre-o-podcasting-portugus.html

Bottomley, A. (2015). Podcasting: A Decade in the Life of a "New" Audio Medium: Introduction. Journal of Radio \& Audio Media, vol. 22, pp. 164-169.

Castro, G. (2005). Podcasting e consumo cultural. Revista da Associação Nacional dos Programas de PósGraduação em Comunicação, e-compos, 53, 1-18.

Cohen, N., e Arieli, T. (2011). Field research in conflict environments: Methodological challenges and snowball sampling. Journal of Peace Research, vol. 48, pp. 423-435.

Cordeiro, P. (2014). Rádio e Internet: novas perspetivas para um velho meio. Actas do II Congresso Ibérico de Comunicação na Covilhã - Universidade da Beira Interior.

Crofts, S., Fox, M., Retsema, A., Williams, B. (2005). 'Podcasting: A New Technology in Search of Viable Business Models', First Monday, 10(9).

Hammersley, B. (2004). Audible Guardian. https://www.theguardian.com/media/2004/feb/12/broadcasting.digitalmedia

Llinares, D., Fox, N. e Berry, R. (2018). Introduction: Podcasting and Podcasts - Parameters of a New Aural Culture. Em Llinares, D., Fox, N. e Berry, R (Ed.), Podcasting. New Aural Cultures and Digital Media. (Edição, volume, pp. 1-13). Palgrave Macmillan

Markman, K. (2015). Considerations-Reflections and future research. Everything old is new again: Podcasting as radio's revival. Journal of Radio \& Audio Media, vol. 22, pp. 240-243.

Markman, M. e Sawyer, C. (2014). Why pod? Further explorations of the motivations for independent podcasting. Journal of Radio \& Audio Media, vol. 21, pp. 20-35.

McGowan, M. (2010) The Unexplored New Medium: Recent Trends in Podcast Advertising. The Elon Journal of Undergraduate Research in Communications. 1(2), pp. 97-111.

Morgan, J. (2015). How Podcasts Have Changed in Ten Years: By the Numbers, Medium. https://medium.com/@slowerdawn/how-podcasts-have-changed-in-ten-years-by-the-numbers720a6e984e4e\#.cdo8de3mx

Portela, P. (2011). Rádio na Internet em Portugal - A abertura à participação num meio em mudança. Vila Nova de Famalicão, Edições Húmus. 
Reis, Ana Isabel (2018). O áudio invisível: uma análise ao podcast dos jornais portugueses. Revista Lusófona de Estudos Culturais. 5(1). pp. $209-225$.

Spinelii, M. e Dann, L. (2019). Podcasting. The Audio Media Revolution. Bloomsbury Academic.

Vanassi, G. (2007). Podcasting como processo midiático interativo. Monografia. Universidade de Caxias do Sul

\section{Anexo:}

Esquematização de Inquérito Online

Título: "Caracterização do podcaster português"

Objetivo: Realizar um retrato dos produtores de podcast em Portugal, retratando, para além da sua idade, género e formação, a origem dos formatos e ambições para a atividade de podcasting.

Data de realização: 23 de Outubro de 2018 a 4 de Fevereiro de 2019.

Plataforma: Google Forms

Modelo de distribuição: Envio pessoal aos produtores e distribuição nos grupos de podcasters portugueses no Facebook

Número de questões: 38

(página 1 "Do podcaster" - 4 perguntas)

- Alguma vez fez rádio antes de ter começado a realizar o podcast?

(Resposta seleção: Sim, Não)

- Se sim em que contexto?

(Resposta múltipla: Rádio universitária, Rádio local, Rádio de cobertura regional ou nacional, Rádio Online, Durante um estágio curricular ou profissional numa rádio, Em contexto académico (ex-aulas ou curso de rádio)

- Em que ano começou a produzir podcasts?

(Resposta seleção com todos os anos entre 2004 a 2019)

- Em quantos formatos participa regularmente?

(Resposta seleção: Um formato, dois formatos, três formatos, mais de três formatos) 
(página 2 "Do podcast"- 10 perguntas, aqui com referencial ao programa ao qual dedica mais tempo)

- Com que frequência lança um novo episódio?

(Resposta seleção: Diariamente, Semanalmente, Quinzenalmente, Mensalmente, Ocasionalmente, O meu podcast não está activo neste momento)

- Consegue manter a frequência regular de lançamento dos novos episódios?

(Resposta seleção: Sim, Quase Sempre, Não)

- Em que plataformas tem o seu podcast?

(Resposta múltipla: Soundcloud, Mixcloud, iTunes, Spotify, Youtube, Outra/s: Qual/is?)

- Onde costuma gravar o seu podcast?

(Resposta múltipla: Em casa, Num estúdio (que não o de uma rádio), Numa rádio, Na rua, Num local público)

- Utiliza material de gravação profissional no seu podcast?

(Resposta seleção: Sim, Não, Não sei)

- Qual a categoria do seu podcast?

(Resposta múltipla: Arte, Negócios, Comédia, Ensino, Jogos e passatempos, Governo e organizações, Saúde, Infantil e familiar, Música, Notícias e Política Religião e espiritualidades, Ciência e medicina, Sociedade e cultura, Desporto e lazer, Tecnologia, TV e cinema).

- Como classificaria o seu podcast?

(Resposta múltipla: Podcast de entrevista, Podcast de debate (conversa) sempre com os mesmos intervenientes, Podcast de debate (conversa) com intervenientes diversos, Monólogo, Storytelling, Áudio de um videocast, Podcast de divulagação musical, Outro/Quais).

- O seu podcast é emitido ou produzido para alguma rádio?

(Resposta seleção: Sim, Não)

- O seu podcast é emitido ou produzido para algum órgão de comunicação social (que não uma rádio)?

(Resposta seleção: Sim, Não)

- O seu podcast tem (assinale todas as opções válidas):

(Resposta múltipla: Site próprio, Perfil de Facebook, Perfil de Instagram, Conta de Twitter, Email público (para poder receber feedback), Financiamento por patronos (tipo Patreon). 
(página 3 "Das motivações do podcaster" - 6 perguntas)

- Lancei um podcast porque queria experimentar rádio:

(Resposta em escala linear: 1 - Não, 6 - Totalmente)

- Realizar o meu podcast é uma forma de me realizar pessoalmente:

(Resposta em escala linear: 1 - Não, 6 - Totalmente)

- Lancei o meu podcast porque queria chegar a uma determinada audiência:

(Resposta em escala linear: 1 - Não, 6 - Totalmente)

- Lancei o podcast também para me divertir:

(Resposta em escala linear: 1 - Não, 6 - Totalmente)

- Motivos financeiros levaram-me a lançar o podcast?

(Resposta em escala linear: 1 - Não, 6 - Totalmente)

- Sinto que o meu podcast é algo único no panorama do podcasting português:

(Resposta em escala linear: 1 - Não, 6 - Totalmente)

(página 4 "Das audiências" - 3 perguntas)

- No último mês, quantos ouvintes teve, em média, cada episódio do seu podcast?

(Resposta aberta)

- As audiências estão de acordo com as suas expectativas?

(Resposta em escala linear: 1 - Não estão de acordo com as expectativas, 6 - Estão totalmente)

- Qual seria para si um "número bom" de ouvintes?

(Resposta aberta)

(página 5 "Das parcerias" - 2 perguntas)

- Tem alguma parceria remunerada no seu podcast?

(Resposta seleção: Sim, Não) 
- Se respondeu sim, quantas parceiras tem no seu podcast:

(Resposta seleção: Uma, duas, três, outra opção)

(página 5 "Da publicidade" - 5 perguntas)

- Tem publicidade no seu podcast?

(Resposta seleção: Sim, Não)

- Se respondeu sim, a quantas marcas/produtos diferentes o seu podcast faz normalmente publicidade remunerada?:

(Resposta seleção: Uma, outra opção)

- Se não, estaria disposto a ter publicidade no podcast:

(Resposta seleção: Sim, Não, Não penso nisso)

- Se respondeu não, porque não estaria disposto a ter anunciantes no formato?

(Resposta múltipla: Não vejo interesse, Receio de perder independência, Ia ser maçador para os ouvintes, Não ganharia nada, Não pretendo fazer dinheiro com o podcast)

- Quanto acharia justo um anunciante pagar por um spot de 20 segundos no início de um episódio do seu podcast?

(Resposta aberta: __ euros)

(página 6 - 8 perguntas)

- Já pensou terminar com a produção do seu podcast?

(Resposta seleção: Sim, Não, Não sei)

- Se pudesse teria o podcast como actividade permanente, única e remunerada?

(Resposta seleção: Sim, Não, Já tenho o podcast como actividade permanente, única e remunerada, Não sei)

- O seu podcast tem mais alguma pessoa com responsabilidade sobre o formato?

(Não, Sim mais uma pessoa, Sim mais duas pessoas, Sim mais três pessoas, Sim, mais quatro ou mais pessoas)

- Se hoje tivesse de entregar um CV, consideraria o seu podcast como currículo?

(Resposta seleção: Sim, Não, Não sei)

- Qual o seu género?

(Resposta seleção: Masculino, Feminino, Neutro, Prefiro não responder) 
- Qual a sua idade?

(Resposta aberta: anos)

- Profissão (por favor seja preciso e evite respostas como Função Pública)

(Resposta aberta)

- Qual o grau máximo de escolaridade completo?

(Resposta seleção: Menos do que o $9^{\circ}$ ano completo, Ensino Básico - $9^{\circ}$ Ano completo, Ensino Secundário - $12^{\circ}$ Ano Completo, Licenciado, Mestre, Doutorado, Prefiro não responder) 